\title{
Gas and Shocks in Barred Galaxies
}

\author{
Peter Teuben \\ Astronomy Department, University of Maryland, College Park, MD \\ 20742
}

\begin{abstract}
A review of the dynamics of the dissipative gas component in barred galaxies is given. The formation of large scale galactic shocks is described, and its implications for star formation and global mass inflow are discussed. Different approaches for modeling the gas will be contrasted, and it will be shown they all produce the same basic morphological picture. However, they do differ in their estimates of mass inflow rates. Some possible directions for future research are indicated.
\end{abstract}

\section{Introduction}

The focal problems in hydrodynamics of barred galaxies are large scale shocks and mass inflow with star formation. Large scale shocks in barred galaxies have been predicted by theory for decades (e.g. Prendergast 1962) but observations have taken a long time to confirm this. The study of periodic orbits came at a later date (e.g. Contopoulos \& Papayannopoulos 1980) and the connection between periodic orbits and gas flow (see also, e.g., Sanders, van Albada \& Teuben 1983) culminated in a two-part paper by Athanassoula (1992a,b).

Earlier studies of the gas flow in barred galaxies (e.g. Sorensen et al. 1976, Sanders \& Huntley 1976, Roberts et al. 1979) concentrated on finding 2D quasistationary steady-state solutions to the gas flow (due to dissipation and torques in a quadrupole potential an exact steady-state is not possible!) and explaining the beautiful "straight offset dust lanes" in terms of large-scale hydrodynamic shocks. Most codes had to apply artificial methods to prevent unphysically high gas inflow rates.

Simplified quasi-particle hydro calculations by Simkin. et al. (1980) showed for the first time how bars can drive gas into the nucleus, although their models required an ILR to obtain a large inflow rate of about $0.1 M_{\odot} \mathrm{yr}^{-1}$. Subsequently Prendergast (1983) noted that the gas inflow rate was not a well determined quantity at all, and that quite different answers could be obtained from different methods to simulate the gas flow.

More recent simulations, notably SPH-based, concentrate on time-evolution and use the obvious gas-inflow as an effective fueling agent to the central regions of AGN-type galaxies (see, e.g., Shlosman et al. 1989, Friedli \& Benz 1993, Wada \& Habe 1995). Simulations by Barnes \& Hernquist (1991) showed that the torque during a galaxy-galaxy encounter was followed by a strong response of the disk, which subsequently brought in most of the gas to the nucleus. Most notably they showed that the main agent responsible for inflow was the gravitational 
torque, not the hydrodynamical (gas) torque. Note that the bar induced by an encounter is transient and usually not as strong as those in "classical" barred galaxies.

Excellent recent reviews on these matters can be found in Prendergast (1983), Sellwood \& Wilkinson (1993), Phinney (1994), and Athanassoula (1994).

\section{Theory}

Although the true interstellar medium is a "multi-phase stew" (Toomre 1977), most research (but see e.g. Chiang \& Prendergast 1985, Jog \& Solomon 1984 and references therein) has focused on the single-fluid approximation, simply ignoring other phases depending on the kind of study. The equations then to be solved are the single-fluid equations of hydrodynamics, written in Lagrangian form as:

$$
\begin{aligned}
\frac{d \rho}{d t} & =-\rho \nabla \cdot \mathbf{v} \\
\rho \frac{d \mathbf{v}}{d t} & =-\nabla P-\rho \nabla \Phi+\cdots
\end{aligned}
$$

where $\cdots$ represent often ignored additional force terms, such as viscosity, magnetic fields, etc. This set of equations has to be closed with an energy equation and equation of state. Frequently an isothermal gas with an equivalent velocity dispersion (sound speed) of order $10-20 \mathrm{~km} \mathrm{~s}^{-1}$ is taken.

There have been several methods to simulate the ISM, which we broadly divide into three major categories.

\subsection{Analytical Solutions}

Although the fluid equations do not generally lend themselves to analytical solutions in astrophysically relevant potentials, some analytical approaches have been successful. In the limit of a weak bar, Wada (1994) and, independently, Lindblad \& Lindblad (1994) have shown that adding a restoring damping force term to epicyclic orbits mimics the fluid equations which can be solved analytically. This damped-orbit gas is non-self-gravitating. The general 90-degree phase change across the major Lindblad resonances was also well understood (see also Sanders \& Huntley 1976).

In this context we should also mention the 1-dimensional quasi-analytical solutions Roberts et al. (1979) obtained of gas flow in spiral and barred potentials. They neglected pressure forces perpendicular to the flow lines and could thus numerically integrate along the flow lines with very high spatial resolution.

\subsection{Fluid Dynamics}

We differentiate between solving the fluid equations grid-based (CFD, often on an Eulerian grid) and particle-based (SPH, Lagrangian). In addition, there are the "Fluid-in-Cell" codes, such as the beam scheme, a bit hard to place in all but a category by itself.

One of the earliest investigations used a Fluid-in-Cell approach (Sorensen et al. 1976, see also Berman et al. 1979). These simulations showed the large scale shocks for the first time, as predicted by Prendergast (1962) and have been 
the benchmark in this field for a long time. The beam scheme code (Sanders \& Prendergast 1974, later extensively used by the Florida group, see e.g. England et al. 1990) simulates gas by placing density weighted delta-function beams in velocity space and moving fractions of gas to neighboring cells, the time-step controlled by a Courant condition that no gas shall move too fast. In some sense the beam scheme code is also a "Fluid-in-Cell" code. Although a tractable and fast code, the beam scheme's limited resolution and high numerical viscosity has limited applications.

Solving the hydro equations $(1,2)$ is relatively straightforward, but simple solutions to the hydro equations quickly show their limitations. First order Euler methods suffer from roundoff at low resolution (e.g. Mulder 1986), and second order implicit and explicit schemes have been developed which were much more successful. Implicit methods can also be used to search for "steady-state" solutions. Modern improvements include flux splitting schemes (FS2, see van Albada 1981, Athanassoula 1992b) as well as piecewise parabolic methods (PPM, see Piner et al. 1995). On cartesian grids the resolution in the nuclear region is not very large, although regridding techniques have been used to improve this (van Albada 1981). In the recent PPM simulations by Piner et al. (1995) a polar grid was used (see also Matsuda et al. 1987) with a resolution down to 2 pc near the inner $(100 \mathrm{pc})$ boundary. In these simulations the ring formation was quite prominent as long as an ILR was present and the $x_{2}$ orbital family is extended enough in the bulge/bar region (Figure 1).

Smooth particle hydrodynamics associates fluid properties with extended particles. The fluid equations are solved on a Lagrangian grid, moving with the individual particles (Gingold \& Monoghan 1977, Lucy 1977). Again, it took a while until the method was applied to the dynamics of barred galaxies. Shlosman et al. (1989) found large inflow associated with the presence of a bar. Wada \& Habe (1992) calculated the response of a selfgravitating SPH gas disk from an imposed oval potential (they actually studied galaxy-galaxy interaction induced gas inflow). For models with an ILR and initial gas-to-stellar mass ratios greater than $10 \%$ the (central ILR) gas ring becomes unstable, collapses and eventually (on a dynamical timescale) most of the gas is driven to the nucleus.

\subsection{Quasi-Particle Simulations}

Sticky particles have been frequently used to simulate and study the dynamical behavior of the ISM. These codes view the ISM as a set of finite size ballistic cool dense clouds (velocity dispersion $\approx 5-10 \mathrm{~km} \mathrm{~s}^{-1}$ ) which collide inelastically. The rules for exchanging energy and momentum during a collision are somewhat adhoc, but reflect the general ideas we have about interactions between GMCs (see also Combes \& Gerin 1985). Additional physics, such as fission, fusion and mass exchange, have been added too. Most of these codes are not self-gravitating. A clear advantage of these codes is that the qualitative effect of certain physical characteristics (e.g. viscosity) can be modeled and understood. The first papers (Miller, Prendergast \& Quirk 1970) concentrated on spiral structure, but Schwarz $(1981,1984)$ studies the formation of rings (ILR, OLR) in ovally distorted potentials (see also Byrd et al. 1994, Palous, these proceedings) and also showed the formation of shocks/density enhancements along the leading edge of 


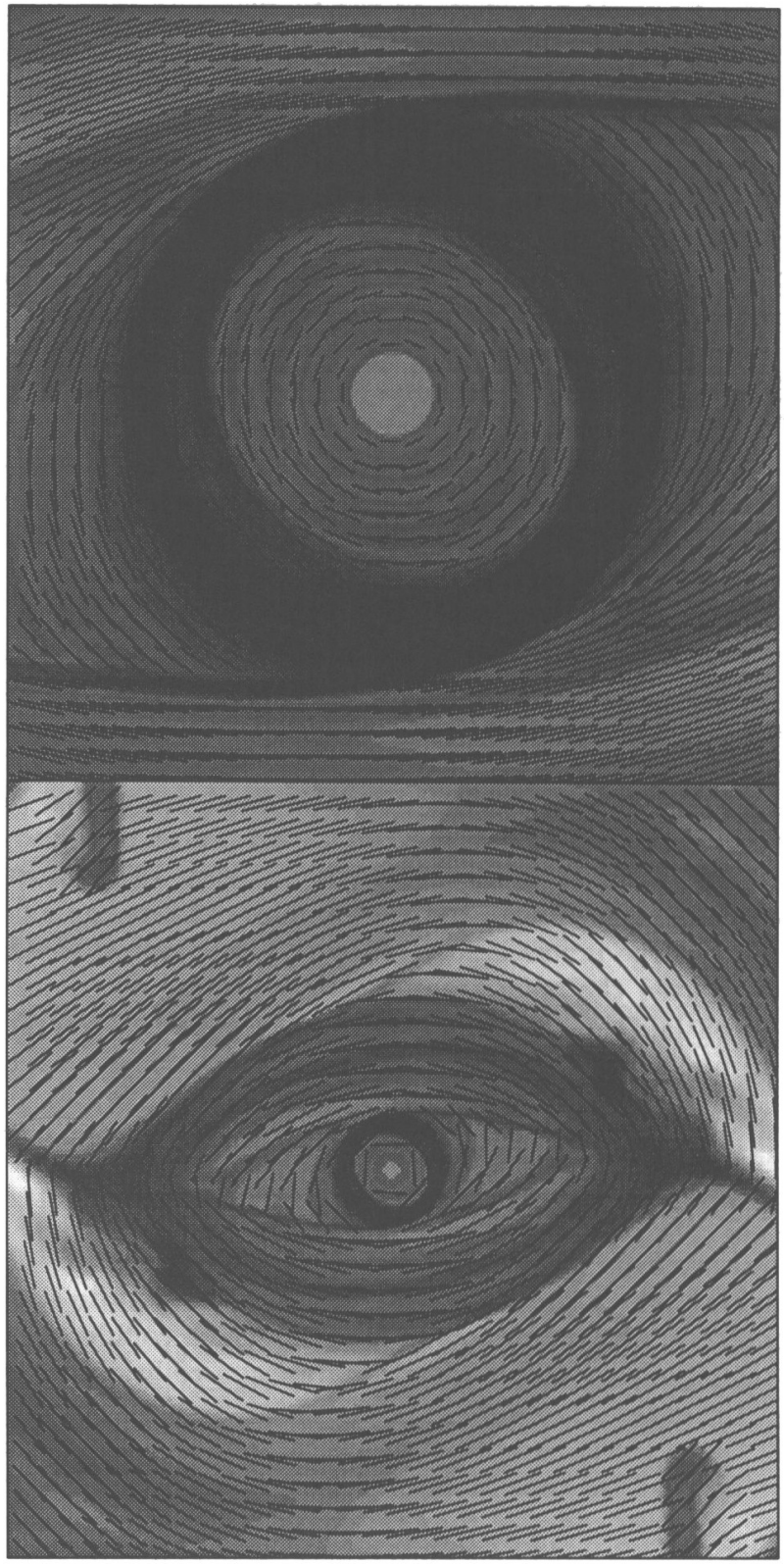

Figure 1. Gas density and flow velocities from Piner et al. (1995). Flow is counter clockwise. The central region is from -1 to $1 \mathrm{kpc}$, the large scale is from -6 to $6 \mathrm{kpc}$ (the bar has a radius of $5 \mathrm{kpc}$, corotation is at $6 \mathrm{kpc}$ ). 
the bar. Gas inflow rates, large enough to fuel AGN, have also been obtained with such codes (Fukunaga \& Tosa 1991).

\subsection{Viscosity}

Various processes in the ISM, such as GMC collisions, turbulent gas motions, star formation, supernovae explosions, and magnetic fields, could possibly be modeled effectively by a bulk viscosity (e.g. Sanders 1977, Wada 1994), since the molecular viscosity is much smaller. Some codes (e.g. beam scheme) have a numeric viscosity which for the right choice of grid and/or time-step could mimic the bulk viscosity of the ISM. In other codes viscosity can be added artificially, sometimes needed to stabilize shock fronts (e.g. SPH, see also Hernquist 1993). Suffice it to say that as long as we don't understand the effective viscosity of the ISM, some of the issues concerning the merits of the various codes and their results will not be resolved.

\section{Results}

All simulations show that the gas is severely redistributed in the bar region of quasi-stationary solutions. Little gas is found perpendicular to the bar (near the stable Lagrangian points $\mathrm{L}_{4}$ and $\mathrm{L}_{5}$ ). Most of the gas can be found in two narrow lanes, which appear to be the loci of strong hydrodynamic shocks.

The shapes of these shocks appear to be related to certain characteristics of the $x_{1}$ family of periodic orbits, in particular the curvature of the orbits at their apocenter and existence of (retrograde) loops (see Athanassoula 1992b).

When the potential allows an ILR (requiring a combination of a centrally concentrated mass distribution, pattern speed not too high and a bar not too strong) and the $x_{2}$ orbital family is fairly extended, the gas within the ILR is on fairly circular orbits and the shocks are offset from the bar.

\subsection{Shocks}

The large scale shocks on the leading edge of the bar have been found in a large number of very different kinds of simulations, all the way from sticky particles, to SPH and grid-based CFD. Shallow water theory, as explained in Prendergast (1983) is a novel approach to explain the shocks and their location. Athanassoula (1992b) combines orbit theory and orbit crowding (looping) to explain the shocks.

\subsection{Athanassoula's 1992 survey}

A landmark survey was the 200-odd experiments Athanassoula performed using van Albada's FS2 code (Athanassoula 1992a,b). We summarize the results:

- there are two kinds of shapes of dust lanes: straight and curved

- an ILR is needed to get straight shocks, in fact, the $x_{2}$ orbital family must be present over a significant portion of the bulge.

- strong shear along the strong shocks probably prohibits star formation, which is thus mainly found near the ends of the bar (cf. the shock focusing model proposed by Roberts et al. 1979) and in the nuclear region. 
- the pattern speed is such that the ratio of the Lagrangian radius to that of the bar is $1.2 \pm 0.2$.

- gas flow associated with the shocks implies inflow. The angle averaged inflow is only a few $\mathrm{km} \mathrm{s}^{-1}$, despite large local variations of $100-200 \mathrm{~km} \mathrm{~s}^{-1}$.

- thin and strong bars, or those that rotate slow enough to have no ILR, feed their gas much faster into the nuclear region.

- weak bars support either no shocks or curved shocks with their concave side towards the bar major axis, again on the leading edge of the bar.

\section{3. $\quad$ Fitting mass models}

One of the aims of detailed hydrodynamic simulations is to determine the mass distribution of barred galaxies. It has long been obvious that the classical approach of a long slit spectrum is not sufficient to determine the mass distribution of non-axisymmetric galaxies. Actually, even two-dimensional velocity fields of "axisymmetric" galaxies have frequently shown that the 1-dimensional approach is insufficient: warps (M83), hidden ovals (M100), and complete asymmetries can cause significant disturbances to the velocity field.

The first major step was the work by Sanders \& Tubbs (1980), applied to the galaxy NGC 5383. Although they did suffer from relatively low resolution (data as well as models) and ambiguous identification of the dust lanes with the large scale shocks, they were able to reproduce the characteristic S-shaped velocity contours. Their best matches occurred for rapidly rotating strong bars, with a centrally concentrated mass distribution (in order to get an $\mathrm{ILR}^{1}$ ). Later attempts by Duval \& Athanassoula (1983), where new photometric data were used to constrain the model fitting, did not significantly improve the fit, suggesting that the relatively low resolution of the beam scheme code may have been the limiting factor.

Slight failures in fitting models and observations have also been attributed (see e.g. Contopoulos et al. 1989, Ball 1993) to ignoring the spiral structure in the model potential. In the ongoing study by Lindblad, Lindblad and Athanassoula (these proceedings) the model fits improve when a spiral potential is added to that of the bar, suggesting that spiral arms form an important component of the galaxy.

\section{Comparison studies}

Comparative studies are important to understand the qualitative and quantitative differences between how the various methods handle shocks and inflow. Moreover, there is the astrophysical question: which method best describes the interstellar medium? To quote Prendergast (1962) "it is unclear what to assume for the equation of state"!

Athanassoula (1994) already noted that detailed comparisons between sticky particles techniques and grid-based CFD techniques become a very laborious and

\footnotetext{
${ }^{1}$ Remarkably, their standard model was later shown not to contain an ILR.
} 
frustrating exercise, having to consider the detailed balance between the necessary gas-recycling and gas inflow. This may in part also be due to inadequacies of the codes.

A comparison between SPH and CFD codes is complicated by the fact that they often don't use the same potential, if used with non-selfgravitating gas. Although generally reported as being very different, a nice counter example is shown by the non-selfgravitating SPH simulations of Ann \& Kwon (these proceedings). Qualitatively these simulations look very much like the strong shock cases as shown in the CFD simulations of e.g. Athanassoula (1992b) and Piner et al. (1995). One obvious difference is the ILR gas ring, which is slightly leading from one perpendicular to the bar ( $\mathrm{cf}$. the sticky particles simulations by Combes \& Gerin 1985). This is something that could be easily confirmed with observations. Mulder (1986) compared grid-models (see also van Albada 1981, van Albada et al. 1982). Matsuda et al. (1987) compared the gas flow in weak ovals, and Lindblad \& Lindblad (1994) compared their analytical solutions to the FS2 code, whereas Wada (1994) did the same with a non-selfgravitating SPH code.

\section{Future}

Matching and fitting models to data can be used not only to determine the internal mass distribution of barred galaxies, but could also be used to provide insight towards the "better ISM descriptor" 2 . With higher resolution and more sensitivity in various gas tracers $(\mathrm{HI}, \mathrm{CO}, \mathrm{H} \alpha, \cdots)$, and possibly stellar absorption velocity fields, this field is likely to become a fruitful endeavor.

Fully 3-dimensional studies will also be needed (see also Fux \& Friedli, these proceedings), as more information on the 3-dimensional structure of bulges becomes available in the future. For example, the apparent difference in tilt between the galactic HI disk and bulge (see discussion in Liszt \& Burton 1996) in the inner parts of our Galaxy needs to be explained.

Modeling the spiral potential outside the region of the bar is probably necessary to obtain better fits (see also Contopoulos et al. 1989). Arguments have been presented that the spiral pattern has a different pattern speed (Sellwood $\&$ Sparke 1988). Further work is currently underway (Sparke, private communication)

Using realistic potentials derived from photometry (e.g. Quillen et al. 1994) will be needed to model real galaxies. This includes asymmetric potentials (Colin \& Athanassoula 1989), as it has become clear that detailed fits will need these.

It has been proposed that double bars (bars within bars, see Shlosman et al. 1989) can drive gas into the nuclear region more efficiently. Although some work on this has been done, we need to understand the behavior of the gas component in these types of galaxies. See also Hasan (these proceedings) for an orbit survey of such potentials.

Hybrid codes are self-consistent combined N-body and (perhaps adaptive, see Shapiro et al. 1994) SPH codes with star formation (Friedli \& Benz 1995).

\footnotetext{
${ }^{2}$ avoiding the term "best" code
} 
They are multi-fluid with heating, cooling, mass loss, etc. For example the code by Chiang \& Prendergast (1985) handles a lot of physics, but only deals with a small patch of the ISM. The future will see these kinds of code merged with the global dynamics and be able to model the ISM in great detail, and perhaps discrepancies in gas inflow rates.

Acknowledgments. I am grateful to E. Athanassoula, J. Stone, S. Vogel and many others for discussions and guidance.

\section{References}

Athanassoula, E. 1992a, MNRAS, 259, 328

Athanassoula, E. 1992b, MNRAS, 259, 345

Athanassoula, E. 1994, in Mass-Transfer induced Activity in Galaxies, I. Shlosman, Cambridge: Cambridge Univ. Press, 143

Ball, R. 1993, ApJ, 395, 418

Barnes, J. E. \& Hernquist, L. 1991, ApJ, 370, 65

Berman, R. H., Pollard, D. J. \& Hockney, R. W. 1979, A\&A, 78, 133

Byrd, G., Rautiainen, P., Salo, H., Buta, R., \& Crocker, D. A. 1994, AJ, 108, 476

Chiang, W. H. \& Prendergast, K. H. 1985, ApJ, 297, 507

Colin, J. \& Athanassoula, E. 1989, A\&A, 214, 99

Combes, F. \& Gerin. M. 1985, A\&A, 150, 327

Contopoulos, G. \& Papayannopoulos, T. 1980, A\&A, 92, 33

Contopoulos, G., Gottesman, S. T., Hunter, J. H. \& England, M. N. 1989, ApJ, 343,608

Duval, M. F. \& Athanassoula, E. 1983, A\&A, 121, 297

Elmegreen, B. G. 1994, ApJ, 425, L73

England, M. N., Gottesman, S. T. \& Hunter, J. H. 1990, ApJ, 348, 456

Friedli, D. \& Benz, W. 1993, A\&A, 268, 65

Friedli, D. \& Benz, W. 1995, A\&A, 301, 649

Fukunaga, M. \& Tosa, M. 1991, PASJ, 43, 469

Gingold, R. A. \& Monoghan, J. J. 1977, MNRAS, 181, 375

Hernquist, L. 1993, ApJ, 404, 717

Jog, C. J. \& Solomon, P. M. 1984, ApJ, 276, 127

Lindblad, P. A. B. \& Lindblad, P. O. 1994, in Physics of the Gaseous and Stellar Disks of the Galaxy, I. R. King, San Francisco: ASP, 29

Liszt \& Burton, B. in Unsolved Problems of the Milky Way, Blitz, L. \& Teuben., P. J., IAU symp. 169 , in press

Lucy, L. B. 1977, AJ, 82, 1013

Matsuda, T., Isaka, H. 1980, Prog. Theor. Phys. 64, 1265

Matsuda, T., Inoue, M., Sawada, K., Shima, E. \& Wakamatsu, K. 1987, MNRAS, 229, 295

Miller, R. H., Prendergast, K. H., \& Quirk, W. S. 1970, ApJ, 161, 903 
Mulder, W.A. 1986, A\&A, 156, 354

Nezlin, M.V. 1994, in Physics of the Gaseous and Stellar Disks of the Galaxy, I. R. King, San Francisco: ASP, 135

Ondrechen, M. \& van der Hulst, J.M. 1989, ApJ, 342, 29

Phinney, E. S. 1994, in Mass-Transfer Induced Activity in Galaxies, I. Shlosman, Cambridge: Cambridge Univ. Press, 1

Piner, B. G. Stone, J. M., \& Teuben, P. J. 1995, ApJ, 449, 508

Prendergast, K. 1962, in Distribution and Motion of Interstellar Matter in Galaxies, L. Woltjer, Benjamin, 217

Prendergast, K. 1983, in Internal Kinematics and Dynamics of Galaxies, E, Athanassoula, Dordrecht: Reidel, 215

Quillen, A. C., Frogel, J. A., Gonzalez, R. A. 1994, ApJ, 437, 1620

Roberts, W. W., Huntley, J. M., \& van Albada, G. D. 1979, ApJ, 233, 67

Sanders, R. H. 1977, ApJ, 217, 916

Sanders, R. H. \& Huntley, J. M. 1976, ApJ, 209, 53

Sanders, R.H., Prendergast, K.H. 1974, ApJ, 188, 489

Sanders, R. H. \& Tubbs, A. D. 1980, ApJ, 235, 803

Sanders, R. H., van Albada, T. S., \& Teuben, P. J. 1983, in Internal Kinematics and Dynamics of Galaxies, E. Athanassoula, Dordrecht: Reidel, 221

Schwarz, M. P. 1981, ApJ, 247, 77

Schwarz, M. P. 1984, MNRAS, 209, 93

Sellwood, J. \& Sparke, L. 1988, MNRAS, 231, 25p

Sellwood, J. \& Wilkinson, A. 1993, Rep. Prog. Phys., 56, 173

Shapiro, P. R., Martel, H., \& Villumsen, J. V. 1994, in Numerical Simulations in Astrophysics, J. Franco et al., Cambridge: Cambridge University Press, 45

Shlosman, I., Frank, J. \& Begelman, M. C. 1989, Nature, 338, 45

Simkin, S. M., Su, H. J. \& Schwarz, M. P. 1980, ApJ, 237, 404

Sorensen, S. A., Matsuda, T., \& Fujimoto, M. 1976, Astrophys. and Space Sci., 43,491

Toomre, A. 1977, Ann. Rev. Astr. Ap., 15, 437

van Albada, G. D. 1981, ApJ, 246, 740

van Albada, G. D. van Leer, B., \& Roberts, W. W. 1982, A\&A, 108, 76

Wada, K. 1994, PASJ, 46, 165

Wada, K. \& Habe, A. 1992, MNRAS, 258, 82

Wada, K. \& Habe, A. 1995, MNRAS, in press

\section{Discussion}

D. Pfenniger: (comment) An important difference between the finite difference and beam scheme codes and the SPH and sticky particle codes is that angular momentum is not conserved locally in the former codes, which is particularly important in self-consistent rotation flow models. 
$J$. Beckman: With any of the theoretical codes now in use, have the theorists predicted any phenomenon which had not been observed, but which was then subsequently observed in a real galaxy?

$P$. Teuben: I can think of the weak shocks that seem time-dependent in the bar region.

A. Bosma: (commenting on the previous question) The radio continuum data of N1097 (Ondrechen \& van der Hulst 1989) showed some weak features in locations that the models also show such weak shocks.

K. Wada: On the analytical model of gas orbits: Lindblad \& Lindblad (1994) also discussed the analytical model, which was almost the same as that in Wada (1994). Especially, they have a nice explanation for the origin of the straight leading shocks in strong bars.

$P$. Teuben: Very nice, it is quite remarkable that applying this linear theory to such a strong bar has resulted in such correspondence to non-linear CFD results!

K. Wakamatsu: Wakamatsu $(1993, \mathrm{AJ}, 105,1745)$ proposed shock wave formation in the rotating gas around $\mathrm{S} 0$ galaxies (known as polar ring galaxies). I showed that the stellar disk potential in certain limited radii of S0 galaxies is deep enough to disturb gas motions severely. I hope you would try to make a hydrodynamical treatment.

$P$. Teuben: I will discuss this with you.

Z. Tsvetanov: What happens in the innermost $1 \mathrm{kpc}$ (or less)? You mentioned that your code has a resolution of around $2 \mathrm{pc}$ in the center.

$P$. Teuben: The potential in our controlled environment becomes very axisymmetric again within the ILR, and little torquing is left. As we now understand some barred galaxies may have inner bars, which could funnel more gas into the center: something we are currently pursuing. In addition, in nuclear rings self-gravity will clearly also play a role here, since the surface densities one finds are generally quite high $\left(1000 \mathrm{M}_{\odot} \mathrm{pc}^{-2}\right)$ (e.g. Wada \& Habe 1992, Elmegreen 1994).

Z. Tsvetanov: Can you distinguish between different shocks in terms of their strength, and what is the typical range of, say, shock velocities?

P. Teuben: The weaker "shocks" (velocity jumps larger than the sound speed over effectively one grid cell) typically jump by perhaps $25-50 \mathrm{~km} \mathrm{~s}^{-1}$, whereas the strong shocks at the leading edge of the bar jump by $100-150 \mathrm{~km} \mathrm{~s}^{-1}$. Also the weaker shocks are transient, and participate in a periodic behavior, unlike the linear offset shocks, which are stationary.

Anonymous: Did you find any shocks in the inner ring?

Teuben: No! At close inspection it's a very tightly wrapped spiral, but no shocks. 